\title{
Analysis of Bonding Properties in Molecular Ground and Excited States by a Cohen-Type Bond Order
}

\author{
BERNHARD DICK \\ Department of Chemistry, University of Pennsylvania, Philadelphia, Pennsylvania, 19104, U.S.A. \\ HANS-JOACHIM FREUND \\ Institut für Organische Chemie der Universität zu Köln, D-5000 Köln 41, Federal Republic of Germany
}

\begin{abstract}
We propose a Cohen-type bond order analysis in terms of orthogonalized atomic basis functions which can be used to analyze NDO wave functions of large organic and metal-organic molecules. It is shown that for small molecules the results gained with this method are in excellent agreement with the same analysis based on ab initio sTO-3G wavefunctions. For large planar aromatic systems these all-valence electron bond orders are found to be a consistent generalization of the $\pi$-bond order. A simple relation between these bond orders and the corresponding covalent bond energies is established. The method can be easily extended to study excited state multiconfiguration wave functions. We present calculations for $\mathrm{C}_{2} \mathrm{H}_{2}, \mathrm{C}_{2} \mathrm{H}_{4}, \mathrm{C}_{2} \mathrm{H}_{6}$, and $\mathrm{Mn}_{2}(\mathrm{CO})_{10}$. The results indicate that the method can be used to discuss the photochemistry of organic and metal-organic compounds.
\end{abstract}

\section{Introduction}

Our understanding of molecular properties has profited quite substantially through the use of certain quantities which, in the rigorous sense of quantum mechanics, are not observables. This implies, however, that there is no unique definition of such quantities. Similar to electronegativity, chemical affinity, bond energies, resonance energies, inductive or mesomeric effects, bond orders are frequently used in the chemical literature to discuss the stability of molecules or molecular fragments.

Due to the above-mentioned ambiguity, several bond order concepts have been proposed. The most commonly applied ones are based on the LCAO formalism [1-5], although there exist more general concepts which do not make use of a particular expansion of the molecular wave function [6]. We study in this paper the applicability of Cohen's bond order [5]. It is a generalization of the well-known Coulson [1] and Mulliken [2] bond order for $\pi$-electron systems and is also closely related to Mulliken's population analysis [3] and the Wiberg index [4]. While in the first two concepts $[1,2]$ the occupation number of a hypothetical diatomic bond orbital determines the bond order between a given pair of atoms, the last two concepts $[3,4]$ aim toward a separation of the total electron density into atomic and diatomic contributions, which are then interpreted as charges and bond orders. Both concepts can be rationalized by a quantum mechanical analysis of the chemical bond via a fragmentation of the total energy into binding energies $[7,8]$. An alternative definition based on the 
eigenvalues of the diatomic charge-and-bond-order submatrix has been introduced by Jug [9]. Very recently this scheme has been applied to excited-state wave functions [10].

Since our main interest is directed to the investigation of larger molecules, especially transition metal compounds and clusters, we are forced to use semiempirical all-valence electron methods based on the NDO approximation [11]. Primarily these calculations yield charge-and-bond-order matrices in a virtual orthogonal basis of atomic orbitals. In order to apply his bond order analysis, Cohen deorthogonalized NDO wave functions using a Löwdin transformation [12]. This procedure, however, contains some degree of arbitrariness within the NDO scheme. In fact, one may ask whether such a transformation is necessary at all in order to apply this bond order analysis. In the course of this paper we, therefore, study the effect of basis set deorthogonalization for a series of selected smaller compounds. In order to ensure the absence of particular NDO artifacts on the bond orders we calculated in a parallel study the corresponding bond orders using minimal basis set $a b$ initio STO-3G wave functions. Subsequently the Cohen bond order analysis is extended to some larger aromatic molecules. In Sec. 3 we apply this method to excited states described by multiconfiguration wave functions. The application to transition metal compounds and their photochemistry is the subject of a subsequent paper [13].

\section{Theory of Cohen's Bond Order Concept}

We start with a brief account of Cohen's bond order concept using his original nomenclature throughout the paper. Generally a bond order $B_{i i^{\prime}}$ may be defined as the ratio of an overlap population $P_{i i}$ to the hypothetical overlap population $P_{i i^{\prime}}^{R}$ of a reference bond:

$$
B_{i i^{\prime}}=P_{i i^{\prime}} / P_{i i^{\prime}}^{R}
$$

If there is only one basis function per center, the natural choice of the covalent reference bond is the equally weighted combination of the two basis functions:

$$
\left|M O_{i i^{\prime}}^{R}\right\rangle=\left(2+2 S_{i i^{\prime}}^{R}\right)^{-1 / 2} \cdot\left(\left|A_{i}\right\rangle+\left|A_{i^{\prime}}\right\rangle\right) .
$$

Here the superscript $R$ denotes that the overlap $S_{i i^{\prime}}^{R}$ has to be taken at some reference distance. This Mo leads to the overlap population $2 \cdot S_{i i^{\prime}}^{R} /\left(1+S_{i i^{\prime}}^{R}\right)$. Therefore, a calculated overlap population $2 \cdot S_{i i^{\prime}} \cdot P_{i i^{\prime}}$ gives the bond order:

$$
\begin{gathered}
B_{i i^{\prime}}=P_{i i^{\prime}} \cdot S_{i i^{\prime}}+P_{i i^{\prime}} \cdot f_{i i^{\prime}}, \\
f_{i i^{\prime}}=S_{i i^{\prime}} / S_{i i^{\prime}}^{R} .
\end{gathered}
$$

If the reference bondlength $R$ is taken equal to the actual bondlength, the "long-range fractor" $f_{i i}$ equals unity and the result is Mulliken's bond order [2]:

$$
B_{i i^{\prime}}^{\text {Mulliken }}=P_{i i^{\prime}}\left(S_{i i^{\prime}}+1\right)
$$


Complete neglect of overlap yields the Coulson bond order [1], which is just the interatomic matrix element of the charge-and-bond-order matrix:

$$
B_{i i^{\prime}}^{\text {Coulson }}=P_{i i^{\prime}} \text {. }
$$

Cohen assumed the reference bond to be an equally weighted combination of two hybrid orbitals [5]:

$$
\left|\mathbf{M O}_{c c^{\prime}}^{R}\right\rangle=\left(2+2 \cdot S_{c c^{\prime}}^{R}\right)^{-1 / 2} \cdot\left(\sum_{i} \alpha_{i}\left|A_{i}\right\rangle+\sum_{i^{\prime}} \alpha_{i^{\prime}}\left|A_{i^{\prime}}\right\rangle\right)
$$

For each of the diatomic symmetry types of the $C_{\infty v}$ point group one reference bond may be defined, e.g., one $\sigma$ bond, two $\pi$ bonds, two $\delta$ bonds, etc. In order to uniquely define the hybridization coefficients $\alpha_{i}$ Cohen postulated that the reference bond should yield the same net atomic populations as the actual LCAO calculation. This leads to the final result for the bond order:

$$
B_{i i^{\prime}}^{\text {Cohen }}=P_{i i^{\prime}} \cdot S_{i i^{\prime}}+P_{i i^{\prime}} \cdot f_{i i^{\prime}} \cdot g_{i i^{\prime}} \text {. }
$$

The factor $g_{i i^{\prime}}$ is a function of the net atomic charges. If the basis set on each atom is orthogonal, it takes the simple form:

$$
\begin{aligned}
g_{i i^{\prime}} & =\alpha_{i} \cdot \alpha_{i^{\prime}} \\
\alpha_{i} & =\left[P_{i i}\left(\sum_{i} P_{j i}\right)^{-1}\right]^{1 / 2} .
\end{aligned}
$$

Here, $j$ runs over all basis functions of the subset to which $i$ belongs. The total $\sigma$-bond order is found by summing all contributions of both $\sigma$ subsets:

$$
B_{\sigma \sigma^{\prime}}=\sum_{i \in \sigma \sigma^{\prime} \in \sigma^{\prime}} B_{i i^{\prime}}
$$

The other $(\pi, \delta$, etc.) bond orders are calculated in a similar way by summing over the appropriate subspace.

Within the framework given above, alternative bond orders could be defined by an alternative definition of the reference hybrids. For example, Cohen's procedure will lead to different reference bond orbitals for the same pair of atoms in the same molecule but different electronic states. In case these reference bonds differ too much, it could make the discussion of photochemical effects more difficult. Fortunately such a situation seems very unlikely when an orthogonal basis is used to evaluate the bond orders (see Sec. $3 F$ ). But the definition of a state-independent reference bond, e.g., derived from standard molecules, is still an option to improve Cohen's bond order where needed.

\section{Results and Discussion}

The problems inherent in the Cohen concept are revealed when results yielded with CNDO wave functions are compared to $a b$ initio results. 


\section{A. Orthogonal and Overlapping Basis Sets}

The Cohen bond order analysis depends on whether an overlapping or nonoverlapping atomic orbital basis is used. Cohen analyzed CNDO wave functions after transformation of the charge-and-bond-order matrix to an overlapping atomic orbital basis via the Löwdin procedure. In this basis the antibonding effects are given a larger weight than the bonding effects. Assuming the reference bond $\mathrm{MO}_{c c^{\prime}}$ and its antibonding counterpart $\mathrm{MO}_{c c^{\prime}}^{*}$ being occupied with $n$ and $n^{*}$ electrons, respectively, the bond order will be:

$$
B_{c c^{\prime}}\left(n, n^{*}\right)=\frac{1}{2}\left[n-n^{*}\left(1+S_{c c^{\prime}}\right) /\left(1-S_{c c^{\prime}}\right)\right] .
$$

If, therefore, the bonding and antibonding orbitals are both doubly filled, a net antibonding effect:

$$
B_{c c^{\prime}}(2,2)=-2 S_{c c^{\prime}} /\left(1-S_{c c^{\prime}}\right),
$$

will result. A numerical value of $S_{c c^{\prime}}=1 / 3$ would be sufficient to yield $B_{c c^{\prime}}=-1$ in such a case. An effect of this kind frequently was observed in the $\sigma$ system of double bonds as, e.g., carbonyl groups. Two bonding and one antibonding orbitals are occupied, leading to a total $\sigma$-bond order of only 0.2 to 0.4 . To overcome this difficulty and the ambiguities connected with the deorthogonalization procedures, we examined the applicability of the Cohen bond order analysis to the charge-and-bond-order matrix directly in the orthogonal basis set. In this case the bond order reduces to:

$$
B_{i i^{\prime}}=P_{i i^{\prime}} f_{i i^{\prime}} g_{i i^{\prime}}
$$

and may be regarded as a generalization of the Coulson bond order.

\section{B. Inner Orbitals}

Chemical intuition suggests that bond orders are a function of valence electrons only and do not depend on whether or not the molecular wave function contains inner orbitals like, e.g., carbon $1 s$ orbitals. The Cohen concept, however, is strongly affected by inclusion of inner orbitals into the basis set from which the reference hybrid will be formed. Since this inner orbital is doubly occupied, the denominator in Eq. (10) increases. Consequently all $g_{i i^{\prime}}$ decrease. Because of the negligible overlap between inner and valence orbitals on two different atoms there is no contribution from Eq. (8) for these orbital pairs. This is exemplified in Tables I(a)-I(e), which contain results of numerical test calculations comparing STO-3G minimal basis set with $\mathrm{CNDO} / 2$ wave functions. Compiled are Wiberg indices, Mulliken overlap populations, and Cohen bond orders. The $\mathrm{CH} \sigma$-bond order, for example, drops from 1.0 to 0.65 in most cases when the core orbital is included. In all cases the bond orders including the core are much smaller than the corresponding bond orders without core. Consequently, in order to apply the Cohen bond order analysis to $a b$ initio wave functions, the inner orbitals have to be projected out to avoid artificial results. Since, however, single-determinant wave functions are invariant against unitary transformation 
among the doubly filled orbitals, there is no unique way to divide the basis set into valence orbitals and inner orbitals. We used two different methods for this purpose depending on whether the bond order analysis was to be performed in an overlapping or an orthogonal basis set.

(i) For an analysis in an overlapping basis the inner orbitals were identified with the lowest energy molecular orbitals resulting from the SCF calculation, one for each non-hydrogen atom. This, to our opinion, most closely resembles the CNDO wave function, since the norm and orthogonality of the remaining MOs are not affected.

(ii) If the bond order analysis was desired in a nonoverlapping basis, after Löwdin orthogonalization of the density matrix the atomic orbitals with the largest coefficient at the $1 s$ basis functions were taken as the $1 s$ inner orbitals and their contribution projected out from the density matrix.

\section{Ground States of Small Molecules}

We now turn to Table Ia-e in detail. We have investigated a set of molecules representing bond configurations typically found for molecules build from atoms of the second row of the periodic table. Intuitively one would expect a bond order of approximately 1.0 for a single, 2.0 for a double, 3.0 for a triple bond, etc. Let us take the $\mathrm{C}-\mathrm{C}$ bonds as an example: Once the core electrons have been projected out, there is fair agreement between $\mathrm{CNDO} / 2$ and sTO-3G results as far as the magnitude and the relative trends are concerned. Clearly the values of the Mulliken overlap population are by far smaller than all bond orders and do not agree with intuitive expectation [14]. The difference between results obtained with orthogonal and overlapping basis sets is in the range of $10 \%$, which is not to be considered serious. As soon as the electronegativity difference between the partners participating in the bonds increases, e.g., in $\mathrm{N}-\mathrm{H}$ or $\mathrm{O}-\mathrm{H}$ bonds, the bond orders based on nonorthogonal atomic orbitals are smaller by $30-40 \%$ than those calculated using orthogonal basis functions. For multiple bonds we find basically the same effect. For the carbon oxygen triple bond in $\mathrm{CO}$ the bond orders are 2.7 (orthogonal basis) and 2.0 (overlapping basis). Again the agreement between the semiempirical and the $a b$ initio minimal basis result is remarkable. Figure 1 shows the correlation between $\mathrm{CNDO} / 2$ and sTO-3G bond orders for both the orthogonal and the overlapping basis sets. Clearly the Cohen bond orders based on orthogonal orbitals fulfill two conditions that are not satisfied with overlapping orbitals. Firstly, the deviation from the unit slope is reasonably small, and secondly the values group in three regions that correlate with our expectation based on chemical intuition. For example, $F_{2}$ has a bond order close to zero in the overlapping basis, while in the orthogonal basis the bond order is reasonable, although it is still the lowest for all molecules studied. Since the concept of a bond order is not free from arbitrariness, we propose here to use the Cohen bond order based on orthogonal all-valence electron wave functions, since we get intuitively appealing results compared with $a b$ initio all-electron wave functions for a variety of small molecules. 
TABLE I. Comparison of bond order data from CNDO/2 and STO-3G minimal basis set calculations. Shown are Wiberg indices (in the orthogonal basis), Mulliken overlap populations (in the overlapping basis), and Cohen bond orders (in both orthogonal and overlapping basis).

\begin{tabular}{|c|c|c|c|c|c|c|c|c|c|c|}
\hline \multirow{2}{*}{$\begin{array}{l}\text { (a) } \\
\text { molecule }\end{array}$} & \multirow[b]{2}{*}{ bond } & \multirow[b]{2}{*}{$\begin{array}{l}\text { Wiberg } \\
\text { CNNO }\end{array}$} & \multicolumn{2}{|c|}{ Mulltken } & \multicolumn{2}{|c|}{ CNDO } & Cohen & & \multirow[b]{2}{*}{ over1." } \\
\hline & & & CNDU & STO-3C & orth. & over1. & orth. & overl. & orth! & \\
\hline \multirow{5}{*}{$\mathrm{C}_{2} \mathrm{H}_{2}$} & $C=c$ & $\sigma$ & & & 0.995 & 0.849 & 0.994 & 0.834 & 0.446 & 0.526 \\
\hline & & $\pi$ & & & 1.000 & 1.000 & 1.000 & 1.000 & 1.000 & I.000 \\
\hline & & $\pi$ & & & 1.000 & 1.000 & 1.000 & 1.000 & 1.000 & 1,000 \\
\hline & & $\Sigma 2.997$ & 0.910 & 0.891 & 2.995 & 2.849 & 2.994 & 2.834 & 2.446 & 2.326 \\
\hline & $\mathrm{C}-\mathrm{H}$ & $\sigma 0.984$ & 0.414 & 0.404 & 0.988 & 0.968 & 0.992 & 0.920 & 0.653 & 0.697 \\
\hline \multirow[t]{5}{*}{$\mathrm{C}_{2} \mathrm{H}_{4}$} & $c=c$ & $a$ & & & 0.988 & 0.956 & 0.978 & 0.826 & 0.434 & 0.527 \\
\hline & & $\pi$ & & & 0.236 & 0.137 & 0.149 & -0.001 & 0.149 & -0.001 \\
\hline & & $\pi$ & & & 1.000 & 1.000 & 1.000 & 1.000 & 1.000 & 1.000 \\
\hline & & $\Sigma 2.058$ & 0.677 & 0.600 & 2.223 & 2.093 & 2,126 & 1.825 & 1.583 & 1. 526 \\
\hline & $\mathrm{C}-\mathrm{H}$ & $\sigma 0.967$ & 0.393 & 0.398 & $0.9 \mathrm{bl}$ & 0.911 & 0.974 & 0.908 & 0.648 & 0.093 \\
\hline \multirow{6}{*}{$\mathrm{C}_{2} \mathrm{H}_{6}$} & $C-C$ & $\sigma$ & & & $0.95 \mathrm{t}$ & 0.966 & 0,925 & 0.802 & 0.406 & 0.514 \\
\hline & & $\pi$ & & & 0.174 & 0.079 & 0.107 & -0.009 & 0.107 & -0.009 \\
\hline & & $\pi$ & & & 0.174 & 0.079 & 0.107 & -0.009 & 0.107 & -0.009 \\
\hline & & $\Sigma 1.060$ & 0.434 & 0.363 & $1 . .298$ & 1.123 & 1.139 & 0.784 & 0.620 & 0.496 \\
\hline & $\mathrm{C}-\mathrm{H}$ & $a 0.979$ & 0.385 & 0.384 & 0.952 & 0.907 & 0.961 & $0.89 \mathrm{l}$ & 0.643 & 0.682 \\
\hline & $C=C$ & $\sigma$ & & & 0.983 & 0.964 & 0.976 & 0.833 & 0.434 & 0.530 \\
\hline \multirow{9}{*}{$\mathrm{CH}_{3}-\mathrm{CH}=\mathrm{CH}_{2}$} & & $\pi$ & & & 0.218 & 0.113 & 0.149 & -0.004 & 0.149 & -0.004 \\
\hline & & $\pi$ & & & 0.976 & 0.987 & 0.986 & 0.997 & 0.986 & 0.997 \\
\hline & & $\sum 1.992$ & 0.680 & 0.610 & 2.177 & 2.063 & 2.111 & 1.827 & 1.569 & 1.523 \\
\hline & $C-C$ & $\sigma$ & & & 0.944 & 0.958 & 0.929 & 0.814 & 0.406 & 0.522 \\
\hline & & $\pi$ & & & 0.149 & 0.056 & 0.096 & -0.012 & 0.096 & -0.012 \\
\hline & & $\pi$ & & & 0.215 & 0.097 & 0.162 & 0.040 & 0.162 & 0.040 \\
\hline & & $\sum 1.044$ & 0.433 & 0.378 & 1.308 & 1.111 & 1.188 & 0.842 & 0.665 & 0.550 \\
\hline & $=\mathrm{C}-\mathrm{H}_{\mathrm{CIS}}$ & $\sigma 0.971$ & 0.407 & 0,411 & 0.466 & 0.919 & 0.977 & 0.916 & 0.651 & 0.698 \\
\hline & $-\mathrm{CH}=$ & $\sigma 0.958$ & 0.391 & 0.408 & 0.959 & 0.889 & 0.975 & 0.905 & 0.650 & 0.691 \\
\hline $\mathrm{CH}_{3} 1 \mathrm{n}$ & plane & 00.982 & 0.393 & 0.386 & 0.955 & 0.923 & 0.962 & 0.897 & 0.644 & 0.686 \\
\hline $\mathrm{CH}_{3}$ out & t plane & 00.971 & 0.381 & 0.382 & 0.948 & 0.894 & 0.958 & 0.885 & 0.641 & 0.677 \\
\hline
\end{tabular}

(b)

\begin{tabular}{|c|c|c|c|c|c|c|c|c|c|c|}
\hline \multirow[t]{5}{*}{$\mathrm{HCN}$} & \multirow[t]{4}{*}{$\mathrm{C} \equiv \mathrm{N}$} & \multicolumn{3}{|l|}{$\sigma$} & 0.977 & 0.384 & 0.985 & 0.554 & 0.490 & 0.348 \\
\hline & & \multicolumn{3}{|l|}{$\pi$} & 0.999 & 0.998 & 1.000 & 1.000 & 1.000 & 1.000 \\
\hline & & \multicolumn{3}{|c|}{$\pi$} & 0.999 & 0.998 & 1.000 & 1.000 & 1.000 & 1.0010 \\
\hline & & 23.006 & 0.655 & 0.737 & 2.974 & 2.380 & 2.984 & 2.553 & 2.489 & 2.347 \\
\hline & $\mathrm{C}-\mathrm{H}$ & 00.977 & 0.405 & 0.398 & 0.986 & 0.948 & 0.986 & 0.905 & 0.647 & 0.691 \\
\hline \multirow[t]{7}{*}{$\mathrm{H}_{2} \mathrm{C}=\mathrm{NH}$} & $\mathrm{C}=\mathrm{N}$ & \multicolumn{3}{|l|}{$\sigma$} & 0.954 & 0.644 & 0.952 & 0.627 & 0.457 & 0.414 \\
\hline & & \multicolumn{3}{|l|}{$\pi$} & 0.259 & 0.146 & 0.203 & 0.052 & 0.203 & 0.052 \\
\hline & & \multicolumn{3}{|l|}{$\pi$} & 0.997 & 0.996 & 0.999 & 0.999 & 0.999 & 0.999 \\
\hline & & $\Sigma 2.059$ & 0.527 & 0.521 & 2.210 & 1.786 & 2.154 & 1.677 & 1.659 & 1.464 \\
\hline & $\mathrm{C}-\mathrm{H}_{\mathrm{cis}}$ & 00.958 & 0.386 & 0.385 & 0.959 & 0.906 & 0.968 & 0.888 & 0.642 & 0.679 \\
\hline & $\mathrm{C}-\mathrm{H}_{\text {trans }}$ & 00.957 & 0.382 & 0.389 & 0.457 & 0.896 & 0.967 & 0.901 & 0.643 & 0.690 \\
\hline & $\mathrm{N}-\mathrm{H}$ & $\sigma 0.947$ & 0.259 & 0.311 & 0.887 & 0.586 & 0.921 & 0.713 & 0.665 & 0.582 \\
\hline \multirow[t]{7}{*}{$\mathrm{CH}_{3}-\mathrm{NH}_{2}$} & $\mathrm{C}-\mathrm{N}$ & \multicolumn{3}{|l|}{$\sigma$} & 0.909 & 0.799 & 0.897 & 0.710 & 0.412 & 0.472 \\
\hline & & \multicolumn{3}{|l|}{$\pi$} & 0.173 & 0.068 & 0.142 & 0.018 & 0.142 & 0.018 \\
\hline & & \multicolumn{3}{|l|}{$\pi$} & 0.155 & 0.067 & 0.103 & -0.008 & 0.103 & -0.0118 \\
\hline & & $\sum 1.049$ & 0.360 & 0.333 & I. 237 & 0.933 & 1.142 & 0.720 & 0.657 & 0.481 \\
\hline & $\mathrm{C}-\mathrm{H}_{1}$ & 00.978 & 0.381 & 0.379 & 0.952 & 0.891 & 0.958 & 0.877 & 0.640 & 0.671 \\
\hline & $\mathrm{C}-\mathrm{H}_{2,3}$ & $\sigma 0.981$ & 0.389 & 0.384 & 0.955 & 0.914 & 0.961 & 0.891 & 0.643 & 0.683 \\
\hline & $\mathrm{N}-\mathrm{H}$ & 00.976 & 0.3111 & 0.344 & 0.928 & 0.729 & 0.947 & 0.794 & 0.669 & 0.639 \\
\hline
\end{tabular}


TABLE I (Continued from previous page.)

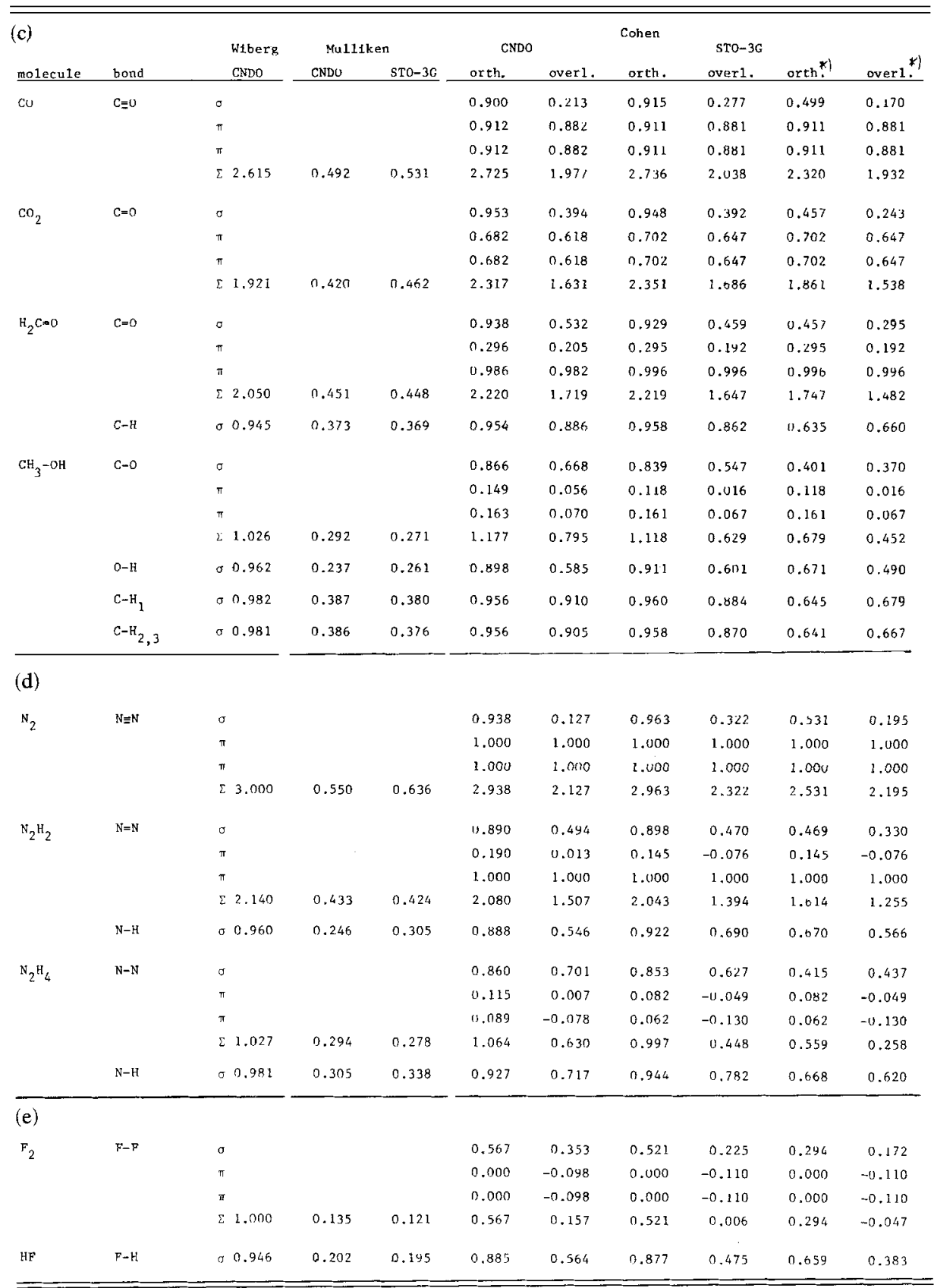

* Including the core $1 s$ orbitals. 




Figure 1. Comparison of Cohen bond orders obtained from CNDO/2 wave functions with those obtained from $a b$ initio STO-3G wave functions. Upper part: orthogonal basis; lower part: overlapping basis.

\section{Ground States of Larger Molecules}

The bond orders for some larger aromatic molecules obtained from CNDO/2 wave functions are shown in Table II. For systems like these bond order discussions in the literature are usually based on Coulson's $\pi$-bond order which is, of course, only meaningful in planar systems. To test whether our concept is consistent with this well-established method we plotted the total bond orders versus the corresponding $\pi$-bond orders (Fig. 2). The correlation coefficient $r=0.995$ indicates very good correlation. The slope of the regression line is, however, significantly larger than one. This implies that the total bond order is not simply the $\pi$-bond order plus a constant, but that the $\sigma$-bond order varies parallel to the $\pi$-bond order for the systems studied here. The Cohen bond order, therefore, turns out to be a useful generalization of the Coulson $\pi$-bond order not restricted to planar $\pi$-electron systems.

Coulson $\pi$-bond orders are found to correlate well with $\mathrm{C}-\mathrm{C}$ bondlengths in unsaturated hydrocarbons. Many linear relationships betwen the $\pi$-bond orders $P_{\pi \pi^{\prime}}$ and the bondlengths $R$ have been suggested to iteratively optimize bondlengths [15]. However, according to Lo and Whitehead [16], the functional dependence $R\left(P_{\pi \pi^{\prime}}\right)$ expected from theory is much more complicated and contains the functions $\partial \beta / \partial R$ and $\partial \gamma / \partial R$, where $\beta(R)$ and $\gamma(R)$ are the resonance and Coulomb integrals of PPP theory. The relation becomes nearly linear only for certain classes of molecules and, as stated by Lo and Whitehead: [16] "the bond distances calculated for molecules other than the calibration molecules 
TABLE II. Cohen bond orders obtained from CNDO/2 wave functions in the orthogonal basis set for some aromatic molecules.




TABLE II (Continued from previous page.)

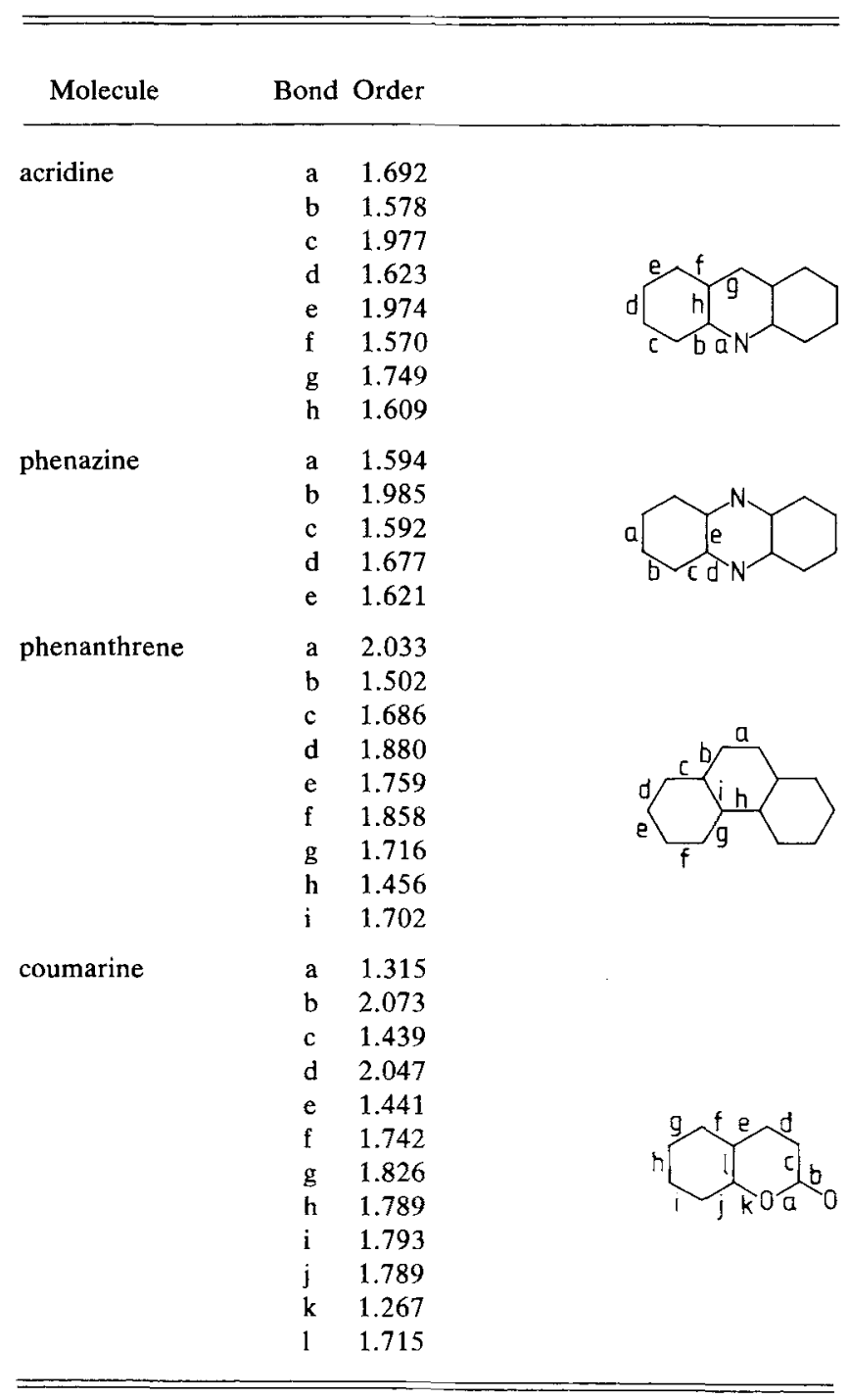

show a wide scatter from such calibration lines." In Figure 3(a) the Cohen C-C bond orders for the molecules listed in Table II are plottted against the corresponding bondlengths, and the same is done for the Coulson $\pi$-bond orders in Figure 3(b). The correlation coefficients are $r=0.890$ for Cohen and $r=0.867$ for $\pi$-bond orders. The Cohen correlation even improves to $r=0.927$ when the molecules with single and triple bonds from Table I are included. This suggests that Cohen bond orders could be used for geometry optimizations in a similar fashion as the $\pi$-bond orders. 


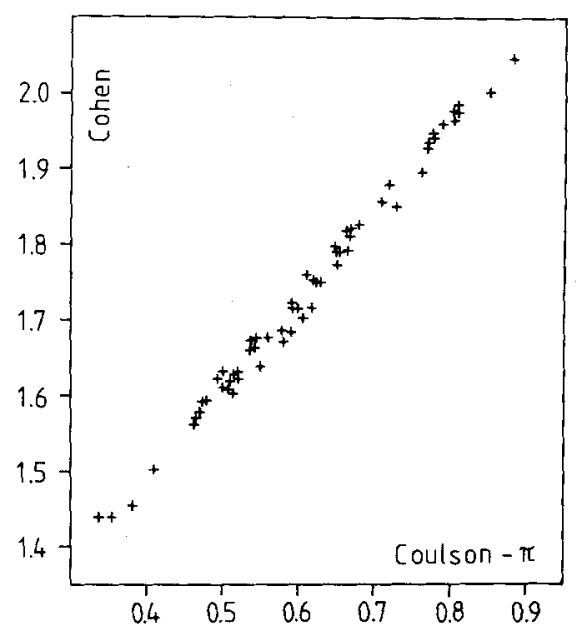

Figure 2. Plot of total Cohen bond orders versus Coulson $\pi$-bond orders for $\mathrm{C}-\mathrm{C}$ bonds. Data are obtained from the molecules shown in Table II.

From Table II it is seen that substitution of ring carbon atoms by nitrogen atoms slightly reduces the bond orders to the neighbors of the nitrogen atom. The corresponding $\pi$-bond orders show, however, nearly no effect. This is because the $\sigma$-bond order sensitively reflects the hybridization state of the nitrogen atom which is different from that of the carbon atom replaced due to the higher electronegativity and the lone pair.
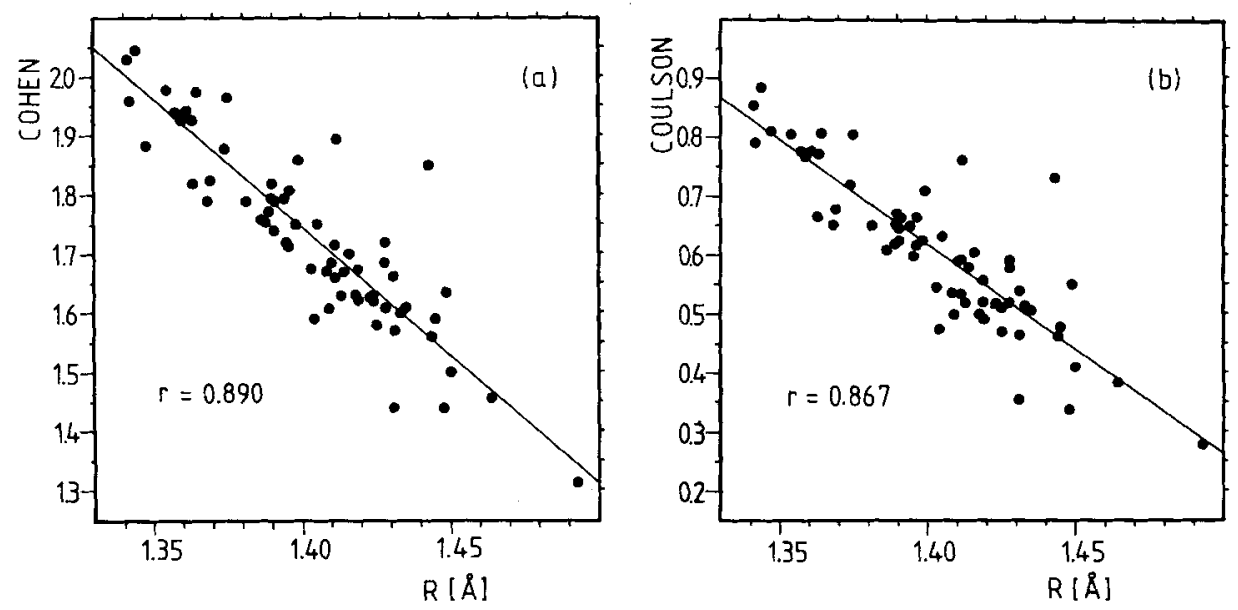

Figure 3. Correlation of: (a) Cohen bond orders and (b) Coulson $\pi$-bond orders for $\mathrm{C}-\mathrm{C}$ bonds versus bondlengths for the molecules listed in Table II. 


\section{E. Bond Orders and Bond Energies}

The stability of molecules is often discussed in terms of bond energies. Values for these quantities may be obtained from thermodynamical data assuming that the total heat of formation with respect to the free atoms is the sum over all bond energies:

$$
\sum_{\text {atoms }} \Delta H_{f}^{\text {gas }}(\text { atom })-\Delta H_{f}^{0}(\text { molecule })=\sum_{\text {bonds }} E(\text { bond })
$$

Using the heat of formation for two hydrocarbons values for $E(\mathrm{C}-\mathrm{C})$ and $E(\mathrm{C}-\mathrm{H})$ could be obtained, but it turns out that the results depend on the hydrocarbons chosen. Therefore, for a large number of hydrocarbons and also for heterocompounds average bond energies have been determined. Differences between these average values and the actual bond energies from thermodynamical data are then used to discuss the stability of a compound.

For multiple bonds these average bond energies are not multiples of the single bond energy, e.g., the $\mathrm{C}-\mathrm{C}, \mathrm{C}=\mathrm{C}$, and $\mathrm{C} \equiv \mathrm{C}$ bond energies are 82.8 , 143.1 , and $187.2 \mathrm{kcal} / \mathrm{mol}$, respectively [17]. The ratio of these values is $1: 1.73: 2.26$, which is quite close to the ratio of the corresponding Cohen bond orders $(1: 1.71: 2.31)$. This suggests a definition for a theoretical bond energy:

$$
E(A-B)=F_{A B} B_{A B},
$$

where $B_{A B}$ is the total bond order and $F_{A B}$ is some scaling factor. If a linear relationship like this were meaningful, the heat of formation for a variety of molecules should have the form:

$$
\Delta H(\text { molecule })-\Delta H(\text { free atoms })=\sum_{\text {bonds }} F_{A B} B_{A B} .
$$

We used this relationship for $\mathrm{C}_{2} \mathrm{H}_{2}, \mathrm{C}_{2} \mathrm{H}_{4}$, and $\mathrm{C}_{2} \mathrm{H}_{6}$ to calibrate values for $F_{\mathrm{CC}}$ and $F_{\mathrm{CH}}$. The values for $F_{\mathrm{NN}}, F_{\mathrm{CN}}$, and $F_{\mathrm{NH}}$ have been obtained from the thermodynamical data for $\mathrm{N}_{2}, \mathrm{HCN}$, and $\mathrm{N}_{2} \mathrm{H}_{4}$, respectively. The results are compiled in Table III.

To test relation [Eq. (17)] values for $\Delta H$ have been calculated for some $\mathrm{C}$, $\mathrm{H}$, and $\mathrm{N}$ containing compounds using the above determined $F$ parameters and compared to the corresponding thermodynamical data (Table IV). The result is encouraging, even for the larger molecules the error is below $5 \%$. Unfortunately the $F_{\mathrm{CO}}$ values obtained from $\mathrm{CO}, \mathrm{CO}_{2}$, and $\mathrm{CH}_{2} \mathrm{O}$ do not agree with each other and are therefore not useful in the above sense. The reason for this behavior, in our opinion, is the large charge separation in these molecules, which gives rise to a pronounced ionic contribution to the bond energy.* Estimation by a point charge model gives contributions of several $20 \mathrm{kcal} / \mathrm{mol}$ for some $\mathrm{CO}$ bonds, while in the above-mentioned $\mathrm{C}, \mathrm{H}$, and $\mathrm{N}$ containing molecules the ionic contributions are always below $1 \mathrm{kcal} / \mathrm{mol}$. A more complete model has to take

\footnotetext{
* More elaborate $\mathrm{CI}$ calculations revealed that the charge distribution for $\mathrm{CO}$ is sensitive to the inclusion of doubly excited configurations into the ground-state wave function. The use of the SCF density matrix may be questionable in this case.
} 


\begin{tabular}{lr}
\begin{tabular}{c} 
TABLE III. Energy fac- \\
tors $F_{A B}$ (in kcal/mol) to \\
$\begin{array}{c}\text { convert Cohen bond orders } \\
\text { into bond energies. }\end{array}$ \\
\hline Bond
\end{tabular} F $_{\mathrm{AB}}$ \\
\hline $\mathrm{C}-\mathrm{C}$ & 62.12 \\
$\mathrm{C}-\mathrm{H}$ & 103.98 \\
$\mathrm{C}-\mathrm{N}$ & 68.01 \\
$\mathrm{~N}-\mathrm{N}$ & 76.92 \\
$\mathrm{~N}-\mathrm{H}$ & 88.92 \\
\hline
\end{tabular}

the ionic bond energies into account properly, too, while the bond orders only contribute to the covalent bond energies.

Table $\mathrm{V}$ gives some representative bond energies for individual bonds derived with the new method. They compare quite well with the thermodynamically determined average values. We, therefore, suggest that differences in bond orders may be directly related to differences in covalent bond energies through the above-described scaling factors.

TABLE IV. Heat of formation from the free atoms for several molecules obtained from thermodynamical data compared to sums over bond energies. The heats of formation of the free atoms from the elements under standard conditions are: $\mathrm{H}, 52.102 \mathrm{kcal} / \mathrm{g}$-atom; C, $170.89 \mathrm{kcal} / \mathrm{g}$-atom; and $\mathrm{N}, 113.0 \mathrm{kcal} / \mathrm{g}$-atom.

\begin{tabular}{lcc}
\hline \hline Molecule & $\mathrm{H}(\exp )$ & $\mathrm{H}(\mathrm{calc})$ \\
\hline $\mathrm{C}_{2} \mathrm{H}_{2}$ & 391.68 & 391.53 \\
$\mathrm{C}_{2} \mathrm{H}_{4}$ & 537.79 & 537.89 \\
$\mathrm{C}_{2} \mathrm{H}_{6}$ & 674.59 & 674.45 \\
$\mathrm{CH}_{3}-\mathrm{CH}=\mathrm{CH}_{2}$ & 820.38 & 813.20 \\
$\mathrm{C}_{6} \mathrm{H}_{6}$ (benzene) & 1318.15 & 1268.94 \\
$\mathrm{~N}_{2}$ & 226.00 & $(226.00)$ \\
$\mathrm{N}_{2} \mathrm{H}_{4}$ & 411.61 & 411.60 \\
$\mathrm{CH}_{3}-\mathrm{NH}$ & \\
$\mathrm{HCN}_{2}$ & 549.90 & 546.67 \\
pyridine & 304.79 & $(304.79)$ \\
naphthalene & 1193.36 & 1186.30 \\
anthracene & 2089.62 & 1988.10 \\
\hline \hline
\end{tabular}


TABLE V. Bond energies for individual bonds (in $\mathrm{kcal} / \mathrm{mol}$ ) $\mathrm{calcu}-$ lated from the corresponding bond orders via Eq. (16).

\begin{tabular}{lccccc}
\hline Molecule & $\mathrm{C}-\mathrm{C}$ & $\mathrm{C}-\mathrm{H}$ & $\mathrm{C}-\mathrm{N}$ & $\mathrm{N}-\mathrm{H}$ & $\mathrm{N}-\mathrm{N}$ \\
\hline $\mathrm{C}_{2} \mathrm{H}_{2}$ & 186.0 & 102.7 & & & \\
$\mathrm{C}_{2} \mathrm{H}_{4}$ & 138.1 & 100.0 & & & \\
$\mathrm{C}_{2} \mathrm{H}_{6}$ & 80.6 & 99.0 & & & \\
benzene & 112.5 & 99.0 & & & \\
$\mathrm{HCN}$ & & 102.5 & 202.3 & & \\
$\mathrm{CH}_{3}-\mathrm{NH}_{2}$ & & 99.3 & 84.1 & 82.5 & \\
& & 99.0 & & & 226.0 \\
$\mathrm{~N}_{2}$ & & & & & \\
$\mathrm{~N}_{2} \mathrm{H}_{2}$ & & & 89.0 & 160.0 \\
$\mathrm{~N}_{2} \mathrm{H}_{4}$ & & & & 82.4 & 81.8 \\
\hline \hline
\end{tabular}

\section{F. Bond Orders in Excited States}

To demonstrate the applicability of Cohen's bond order analysis to excitedstate multiconfiguration wave functions we present results for ethane, ethylene, and acetylene as test examples for covalent single, double, and triple bonds. The excited-state wave functions were generated by configuration interaction among all singly excited configurations from the $\mathrm{CNDO} / 2 \mathrm{SCF}$ ground state. The resulting bond orders for the $\mathrm{C}-\mathrm{C}$ bonds both in the orthogonal and the overlapping basis are presented in Table VI together with the corresponding Wiberg indices.

Each electron excitation is accompanied by a shift of population among orbitals of different bonding character. If the diatomic molecular orbitals are pure bonding $(b)$, antibonding $\left(b^{*}\right)$, or nonbonding $(n)$, each electron removed from (or added to) a bonding orbital will decrease (increase) the bond order by 0.5 . For antibonding orbitals the effect will be of the opposite sign, and in the case of the overlapping basis also of higher weight.

In our examples only the $\pi$ orbitals of acetylene and the out-of-plane $\pi$ orbitals of ethylene may be classified as pure bonding and antibonding, while all other orbitals are mixtures of varying $\sigma, \sigma^{*}$, and $n$ character. The $c 1$ will cause a further mixing of these components, and the resulting bond orders might be helpful in assigning the character of a multiconfiguration wave function in terms of partial $n \rightarrow \sigma^{*}$, etc., nature.

The results obtained in the orthogonal basis are in full agreement with this phenomenological description. In acetylene the HOMO and LUMO are the bonding $\pi_{u}$ and the antibonding $\pi_{\mathrm{g}}$ orbitals. All low-lying states involve an excitation of one electron from $\pi_{u}$, therefore the $\pi$-bond order is decreased by 0.5 . The 
TABLE VI. Cohen bond orders and Wiberg indices for $\mathrm{C}-\mathrm{C}$ bonds in some excited states of $\mathrm{C}_{2} \mathrm{H}_{2}$, $\mathrm{C}_{2} \mathrm{H}_{4}$, and $\mathrm{C}_{2} \mathrm{H}_{6}$ as obtained from CNDO/2-CI calculations.

\begin{tabular}{|c|c|c|c|c|c|c|c|c|c|c|c|}
\hline \multicolumn{3}{|c|}{ state } & Wiberg & \multirow{2}{*}{\multicolumn{3}{|c|}{ orthogonal basis }} & \multirow{2}{*}{\multicolumn{2}{|c|}{ Cohen }} & \multirow{2}{*}{\multicolumn{3}{|c|}{ verlapping basis }} \\
\hline \multirow{5}{*}{$\mathrm{C}_{2} \mathrm{H}_{2}$} & & & & & & & & & & & \\
\hline & $1 . \Sigma_{p}^{+}$ & G요 & 2.997 & 0.995 & 1.000 & 1.000 & $\begin{array}{r}\text { sum } \\
2.995\end{array}$ & 0.844 & 1.000 & 1.000 & 2.849 \\
\hline & $1 \Delta_{u}$ & $\pi_{u} \rightarrow \pi_{g}$ & 1.497 & 0.995 & 0.500 & 0.500 & 1.995 & 0.849 & 0.247 & 0.247 & 1.343 \\
\hline & $1 \pi_{g}$ & ${ }_{u} \rightarrow \sigma_{u}^{*}$ & 2.042 & 0.856 & 0.750 & 0.750 & 2.356 & -1.446 & 0.750 & 0.750 & 0.055 \\
\hline & $1 \pi_{11}$ & ${ }_{\mathrm{tu}} \rightarrow \sigma *$ & 2.274 & 0.991 & 0.750 & $0.75 n$ & 2.497 & 0.802 & 0.750 & 0.150 & 2.302 \\
\hline \multirow[t]{5}{*}{$\mathrm{C}_{2} \mathrm{H}_{4}$} & $1 A_{p}$ & GS & 2.058 & 0.988 & 0.236 & 1.000 & 2.223 & 0.956 & 0.137 & 1.000 & 2.093 \\
\hline & $\mathrm{LB}_{3 \mathrm{p}}$ & $5 \pi \rightarrow 7 \pi^{*}$ & 1.426 & 0.988 & 0.416 & 0.500 & 1.904 & 0.956 & 0.262 & $0.12 y$ & 1.347 \\
\hline & ${ }^{18} 3 u$ & $\pi \rightarrow \pi^{*}$ & 0.881 & 0.882 & 0.240 & 0.144 & 1.266 & 0.259 & 0.146 & -0.174 & 0.230 \\
\hline & $1 \mathrm{R}_{1}$ & $40 \rightarrow \pi^{*}$ & 0.971 & 0.786 & 0.235 & 0.500 & 1.522 & 0.680 & 0.138 & 0.129 & 0,948 \\
\hline & ${ }^{2 \mathrm{~B}} \mathrm{~g}_{\mathrm{g}}$ & $\pi \rightarrow 1 \sigma^{\star}$ & 1.208 & 0.830 & 0.236 & 0.500 & 1.566 & -1.419 & 0.138 & 0.500 & -0.782 \\
\hline \multirow[t]{5}{*}{$\mathrm{C}_{2} \mathrm{H}_{6}$} & $\mathrm{IA}_{\mathrm{ig}}$ & $r s$ & 1.060 & 0.951 & 0.174 & 0.174 & 1.298 & 0.966 & 0.079 & 0.079 & 1.123 \\
\hline & $l \mathrm{~F}_{\mathrm{u}}$ & $1 e_{g}+2 e_{u}$ & 1.111 & 0.843 & 0.297 & 0.291 & 1.437 & -0.163 & 0.260 & 0.260 & 0.356 \\
\hline & ${ }^{1 \mathrm{~A}} 1 \mathrm{H}$ & $\operatorname{le}_{\mathrm{g}} \rightarrow 2 \mathrm{e}_{\mathrm{u}}$ & 1.242 & 0.951 & 0.348 & 0.348 & 1.646 & 0.966 & 0.413 & 0.413 & 1.793 \\
\hline & ${ }^{1 A_{2 u}}$ & $1 e_{g}+2 e_{u}$ & 1.189 & 0.928 & 0.341 & 0.341 & 1.610 & 0.888 & 0.400 & 0.400 & 1.689 \\
\hline & $1 E_{g}$ & $\mathrm{le}_{\rho}+3 \mathrm{a}_{\mathrm{g}}$ & 1.126 & 0.948 & 0.223 & 0.223 & 1.393 & 1.100 & 0.049 & 0.049 & 1.197 \\
\hline
\end{tabular}

lowest virtual $\sigma$ orbitals are $4 \sigma_{\mathrm{g}}$ and $3 \sigma_{u}$ (including the $\mathrm{C} 1 s$ orbitals in the numbering scheme), which are of $\mathrm{C}-\mathrm{H}$ antibonding and $\mathrm{C}-\mathrm{C}$ nonbonding character. The state $1 \Delta_{u}$, the low-lying component of the $\pi_{u} \rightarrow \pi_{g}^{*}$ transition, cannot couple with configurations involving $\sigma$-orbital excitation by symmetry. Therefore only the $\pi$-bond order drops by one unit. In the next two states, $1 \Pi_{g}$ and $1 \Pi_{u}$, the $\sigma$-bond orders are only slightly affected in accordance with the $\mathrm{C}-\mathrm{C}$ nonbonding nature of the involved $\sigma$-orbitals. This may be generalized to the low-lying states of ethylene and ethane also, since all $\sigma$ orbitals involved in the low-lying states are mainly of $\mathrm{C}-\mathrm{H}$ bonding, respectively, antibonding, character. The $\sigma$-bond orders are, therefore only slightly lowered, the largest effect being $\Delta B_{\sigma}=-0.2$ for the $1 B_{1 g}$ state of $\mathrm{C}_{2} \mathrm{H}_{4}$. The in-plane $p$ orbitals contribute to some of the $\sigma$ orbitals in a $\mathrm{C}-\mathrm{C}$ antibonding manner, and bonding to some $\sigma^{*}$ orbitals. Therefore the corresponding $\pi$-bond orders ( $\pi$ with respect to the diatomic system $\mathrm{C}-\mathrm{C}$ ) increase. The net effect is for ethane an increase of the total bond order for all low-lying excited states. In $\mathrm{C}_{2} \mathrm{H}_{4}$ this effect partly counterbalances the lowering of the out-of-plane $\pi$-bond order in $1 B_{3 \mathrm{~g}}\left(\pi \pi^{*}\right)$ state. The two $\pi$-bond orders are of comparable magnitude in this state, indicating the possibility for cis/trans isomerization by rotation around the $\mathrm{C}-\mathrm{C}$ bond. In the $1 B_{3 u}$ state the $\pi$-bond order is not lowered by exactly one unit, since a considerable amount of $\sigma \sigma^{*}$ character mixes into this state. The net effect is still a decrease of the bond order by 0.95 .

In the overlapping basis the bonding effects are still treated correctly as seen in the states $\Sigma_{\mathrm{g}}^{+}, \Pi_{\mathrm{g}}, \Pi_{u}$ of $\mathrm{C}_{2} \mathrm{H}_{2}$ and $1 A_{\mathrm{g}}, 2 B_{1 \mathrm{~g}}$ of $\mathrm{C}_{2} \mathrm{H}_{4}$. The antibonding 
contributions are, however, overestimated by at least a factor of 2 . The most drastic effects occur in the $\sigma$-bond orders where a decrease of about 2.4 is observed in the $\Pi_{\mathrm{g}}\left(\mathrm{C}_{2} \mathrm{H}_{2}\right)$ and the $2 \mathrm{~B}_{1 \mathrm{~g}}\left(\mathrm{C}_{2} \mathrm{H}_{4}\right)$ states. A decrease this large is unreasonable in a basis of only singly excited configurations and must be an artefact of the overlapping basis. Two sources of error seem likely: The first is connected with the deorthogonalization scheme itself which is applied to orbitals which have not been made orthogonal during the calculation. Therefore, some of the nondiagonal terms of the density matrix can get a wrong phase factor during the Löwdin procedure. A second, more pronounced effect, seems to be due to the influence of the deorthogonalization procedure on the reference hybrid. The deorthogonalization shifts a large population into the $2 s$ orbitals $\left(\boldsymbol{P}_{2 s 2 s}>2\right.$ possible) resulting in an artificially large coefficient of this orbital in the reference hybrid. If the overlapping basis is used to analyze excited states, a different definition of the reference hybrid is required. The most natural choice seems to be the corresponding reference hybrid from the SCF ground state.

Finally an artefact of the Wiberg index should be mentioned which shows up in the excited-state calculations. This is because the Wiberg index depends on the square of density matrix elements. It is, therefore, not additive if the bonding contributions are distributed over two or more bonding orbitals. As an example consider the $1 \Delta_{u}$ state of $\mathrm{C}_{2} \mathrm{H}_{2}$. Here the total $\pi$-bond order is 1 $(0.5+0.5)$, while the $\pi$-Wiberg index drops from the ground state value of 2 $\left(1^{2}+1^{2}\right)$ to $0.5\left(0.5^{2}+0.5^{2}\right)$. Similar effects arise in the other excited states listed in Table VI as soon as the two orbitals between which excitation takes place contribute to different matrix elements of the density matrix.

\section{G. Application to Transition Metal Complexes. Case Study: $\mathrm{Mn}_{2}(\mathrm{CO})_{10}$}

In this section we present an example for the application of a Cohen bond order analysis to the ground and an excited state of dimanganese decacarbonyle. It is well known [18] that in $\mathrm{Mn}_{2}(\mathrm{CO})_{10}$ two $\mathrm{Mn}(\mathrm{CO})_{5}$ moieties of $C_{4 v}$ symmetry are bound via a metal-metal $\sigma$ bond to yield a staggered binuclear complex of $D_{4 d}$ symmetry. We have presented calculations on this complex in a previous publication [18]. Table VII compares the Wiberg indices previously published for the ground state of this system [18] with Cohen bond orders. For bonds involving metal atoms the decomposition of the bond orders in the $\sigma, \pi$, and $\delta$ increments is also shown.

Compared with free CO (see Table I) the Cohen bond orders in the nonoverlapping basis set show the intuitively appealing trend. In a linear metal-CO linkage the $\mathrm{CO}$ bond order is reduced from about 2.7 to about 2.5 indicating a weaker $\mathrm{CO}$ bond. The corresponding metal-C bond order is by 0.5 units larger than one. The total bonding capability of the carbon atom adds up to four units. This is neither reproduced with the Wiberg indices nor by the Cohen bond orders in the overlapping basis. It is interesting to note that the $\pi$ character of all metal- $\mathrm{C}$ bonds is almost twice as large as the $\sigma$ character. For the axial metal-C interaction the $\sigma$-bond order is even antibonding in the overlapping basis. The 
TABLE VII. Cohen bond orders and Wiberg indices for the ground state and the lowest $\sigma \neg \sigma^{*}$ excited state of $\mathrm{Mn}_{2}(\mathrm{CO})_{10}$. Overl, overlapping basis; orth., orthogonal basis. The wavefunctions were obtained by a CNDO-CI calculation including 200 energy-selected singly and doubly excited configurations according to the method described in Ref. 21.

\begin{tabular}{|c|c|c|c|c|c|c|c|}
\hline & & grour & tate & & $\sigma-\sigma^{*}$ & ited st & \\
\hline Bond & & Wiber? & $\begin{array}{l}\text { Cohe } \\
\text { orth. }\end{array}$ & over1. & Wiberg & $\begin{array}{l}\text { Cohe } \\
\text { orth. }\end{array}$ & overt. \\
\hline$M n-M_{n}$ & $\sigma$ & & 0.538 & 0.735 & & 0.057 & -1.857 \\
\hline & $\pi$ & & 0.003 & -0.245 & & 0.003 & -0.245 \\
\hline & $\pi$ & & 0.003 & -0.245 & & 0.003 & -0.245 \\
\hline & $\delta$ & & 0.000 & -0.028 & & 0.000 & -0.028 \\
\hline & $\delta$ & & 0.000 & -0.028 & & 0.000 & -0.028 \\
\hline & $\Sigma$ & 0.320 & 0.544 & 0.190 & 0.288 & 0.063 & -2.402 \\
\hline $\mathrm{Mn}-\mathrm{C}_{\mathrm{ax}}$ & $\sigma$ & & 0.567 & -0.223 & & 0.633 & -0.247 \\
\hline & $\pi$ & & 0.476 & 0.307 & & 0.476 & 0.307 \\
\hline & $\pi$ & & 0.476 & 0.307 & & 0.476 & 0.307 \\
\hline & $\Sigma$ & 0.788 & 1.520 & 0.391 & 0.971 & 1.585 & 0.368 \\
\hline$M_{12} \sim C_{\mathrm{eq}}$ & $\sigma$ & & 0.631 & 0.165 & & 0.626 & -0.308 \\
\hline & $\pi$ & & 0.409 & 0.236 & & 0.409 & 0.236 \\
\hline & $\pi$ & & 0.446 & 0.290 & & 0.408 & 0.276 \\
\hline & $\Sigma$ & 0.801 & 1.486 & 0.690 & 0.788 & 1.444 & 0.203 \\
\hline$c-o_{a x}$ & $\Sigma$ & 2.171 & 2.469 & 1.923 & 2.173 & 2.475 & 1.933 \\
\hline $\mathrm{C}-\mathrm{O}_{\text {eq }}$ & $\Sigma$ & 2.202 & 2.489 & 1.959 & 2.236 & 2.511 & 1.975 \\
\hline
\end{tabular}

main result for the ground state, however, is the pure $\sigma$ bond between the metal atoms found in the orthogonal basis. As pointed out in our previous paper [18] on binuclear compounds this is the only result in agreement with simple orbital considerations, since $\pi$ and $\pi^{*}, \delta$ and $\delta^{*}$ Mos that can be created from two sets of atomic $3 d$ functions are filled pairwise in a $d^{7}$ system, while only one $\sigma$-bonding orbital is doubly filled. This $\sigma$ orbital is the highest occupied MO in $\mathrm{Mn}_{2}(\mathrm{CO})_{10}$. The lowest unoccupied $d$ orbital is a $\sigma^{*}$ orbital followed by a $\delta$ and $\delta^{*}$ orbital at higher energies [18].

Photochemical studies on the dissociation of $\mathrm{Mn}_{2}(\mathrm{CO})_{10}$ into two $\mathrm{Mn}(\mathrm{CO})_{5}$ moieties have revealed that very probably the excitation between the $\sigma$ and $\sigma^{*}$ metal-metal bond orbitals induces dissociation of the complex [19]. It was assumed that the first weak absorption band in the UV spectrum of $\mathrm{Mn}_{2}(\mathrm{CO})_{10}$ is due to this excitation, since selective excitation of this band is followed by dissociation $[19,20]$. In order to study this aspect of the photochemical behavior of $\mathrm{Mn}_{2}(\mathrm{CO})_{10}$ we have calculated the electronic absorption spectrum using a 
configuration interaction treatment previously applied to study the photochemical behavior of transition metal carbonyls [21]. The Cohen bond order analysis of the lowest $\sigma-\sigma^{*}$ excited state at $3.03 \mathrm{eV}$ is given in Table VII. In the orthogonal basis the results are very reasonable. The $\sigma$-bond order between the metal atoms nearly vanishes indicating dissociation of the metal-metal bond. The metal-C and $\mathrm{C}-\mathrm{O}$ bond orders are only slightly altered. The same analysis using overlapping orbitals overestimates antibonding contributions as already observed with the small molecules. The absolute value of the Wiberg index does not change adequately so that it is hard to judge on the basis of the Wiberg index exclusively. Since the Wiberg index is calculated from the square of the density matrix, it counts the antibonding contributions with the wrong sign. The Cohen bond order analysis performed in a basis of orthogonal atomic orbitals, however, seems to be a useful tool to study the photochemistry of transition metal compounds.

\section{Conclusions}

From this investigation of Cohen's bond order concept we can summarize the following conclusions.

(i) The Cohen bond order analysis yields similar results for CNDO and STO-3G $a b$ initio wave functions. Therefore its basis set dependence is expected to be only small. Before an application to $a b$ initio wave functions, however, the core electron part of the density matrix has to be projected out.

(ii) The Cohen bond order analysis can be performed in an overlapping or an orthogonal atomic orbital basis. The first basis overemphasizes antibonding contributions.

(iii) For planar aromatic molecules Cohen bond orders correlate well with $\pi$-bond orders. They can therefore serve as a generalization not restricted to planar or unsaturated systems. Both Cohen and Coulson $\pi$-bond orders correlate equally well with bondlengths.

(iv) Application of the Cohen bond order analysis to excited-state wave functions gives a chemically appealing picture of which bonds are weakened by electronic excitation. It can therefore serve as a useful tool to the understanding of photochemical processes.

(v) The Cohen bond order analysis is applicable to any method for which a one-particle density matrix in terms of atomic valence orbitals can be defined. It is therefore neither limited to semiempirical methods nor to $\mathrm{SCF}$ wave functions. Application to CNDO-CI wave functions has been demonstrated here, but other CI or MCSCF wave functions could be analyzed in the same way. In view of the present results we recommend to use an orthogonal atomic orbital basis for the bond order analysis in all cases.

\section{Acknowledgments}

One of us (B.D.) gratefully acknowledges a research fellowship from the Deutsche Forschungsgemeinschaft. We also like to thank the Regionales Rechenzentrum, Köln, for providing the necessary computer time. 


\section{Bibliography}

[1] C. A. Coulson, Proc. R. Soc. (London) Ser. A 169, 413 (1939).

[2] R. S. Mulliken, J. Chem. Phys. 23, 1841 (1955).

[3] R. S. Mulliken, J. Chem. Phys. 23, 1833, 2338, 2343 (1955); 36, 3428 (1962).

[4] $\mathrm{K}$. Wiberg, Tetrahedron 24, 1083 (1968). (Wiberg's bond index is defined as: $W_{A B}=$ $\sum_{\mu \in A} \sum_{\nu \in B} P_{\mu \nu}^{2}$, where $\mu$ and $\nu$ run over the orthogonal atomic valence orbitals at the centers $A$ and $B$, respectively.)

[5] I. Cohen, J. Chem. Phys. 57, 5076 (1972).

[6] R. F. W. Bader, Acc. Chem. Res. 8, 34 (1975).

[7] F. Driessler and W. Kutzelnigg, Theor. Chim. Acta (Berlin) 43, 1 (1976); 43, 307 (1977).

[8] V. Barone, G. DelRe, and S. Fliszar, J. Chem. Soc. Perkin II, 1309 (1979).

[9] K. Jug, J. Am. Chem. Soc. 99, 7800 (1977); 100, 6581 (1978); Theo. Chim. Acta (Berlin) 51, 331 (1979).

[10] P. C. Mishra and K. Jug, Theo. Chim. Acta (Berlin) 61, 559 (1982).

[11] (a) J. A. Pople, D. P. Santry, and G. A. Segal, J. Chem. Phys. 43, S129 (1965); (b) J. A. Pople and G. A. Segal, J. Chem. Phys. 43, S136 (1965); (c) J. A. Pople, D. L. Beveridge, and P. A. Dobosh, J. Chem. Phys. 47, 2026 (1967).

[12] P. -O. Löwdin, J. Chem. Phys. 18, 365 (1950).

[13] H.-J. Freund and B. Dick, to be published.

[14] (a) P. R. Smith and J. W. Richardson, J. Chem. Phys. 71, 924 (1967); (b) K. Ruedenberg, Rev. Mod. Phys. 34, 326 (1962).

[15] (a) M. J. S. Dewar and G. J. Gleicher, J. Am. Chem. 87, 685 (1965); (b) K. Nishimoto and L. S. Forster, Theo. Chim. Acta (Berlin) 3, 407 (1965); (c) C. A. Coulson and A. Golebiewski, Proc. Phys. Soc. London 78, 1310 (1961).

[16] D. H. Lo and M. A. Whitehead, Can. J. Chem. 46, 2027, 2041 (1968).

[17] C. T. Mortimer, Reaction Heats and Bond Strengths (Pergamon, New York, 1962), p. 62.

[18] H.-J. Freund, B. Dick, and G. Hohlneicher, Theo. Chim. Acta (Berlin) 57, 181 (1980).

[19] M. Wrighton, Chem. Rev. 74, 401 (1974); Top. Curr. Chem. 65, 37 (1976).

[20] R. A. Levenson, H. B. Gray, and G. P. Caesar, J. Am. Chem. Soc. 92, 3653 (1970).

[21] B. Dick, H.-J. Freund, and G. Hohlneicher, Mol. Phys. 45, 427 (1982).

Received October 13, 1982

Accepted for publication March 2, 1983 